\title{
01.3
}

\section{Метод выделения характерных фаз поведения в сложных сетях, находящихся в режиме перемежающейся обобщенной синхронизации}

\author{
(C) А.А. Короновский ${ }^{1}$, О.И. Москаленко ${ }^{1}$, А.А. Пивоваров ${ }^{1}$, \\ A.E. Храмов ${ }^{2,1}$ \\ ${ }^{1}$ Саратовский национальный исследовательский государственный \\ университет им. Н.Г. Чернышевского \\ ${ }^{2}$ Саратовский государственный технический университет \\ им. Гагарина Ю.А. \\ E-mail: vostochny@list.ru
}

Поступило в Редакцию 14 октября 2016 г.

Предложен метод выделения фаз синхронных и асинхронных интервалов во временных реализациях взаимодействующих хаотических систем, представляющих собой элементы сети со сложной топологией связей и находящихся в состоянии перехода к режиму обобщенной синхронизации. Данный метод позволяет определять продолжительность фаз синхронной и асинхронной динамики, что потенциально дает возможность проанализировать статистические характеристики перемежающегося поведения рассматриваемых систем.

DOI: 10.21883/PJTF.2017.07.44463.16523

Среди известных типов хаотической синхронизации [1], таких как фазовая синхронизация, синхронизация с запаздыванием (lagсинхронизация), полная синхронизация, режим обобщенной синхронизации является одним из наиболее интересных [2-4]. Понятие обобщенной синхронизации изначально было введено для двух однонаправленно связанных осцилляторов [2] и позднее было расширено на случаи осцилляторов с взаимной связью и сложные сети [5-7]. В качестве взаимодействующих осцилляторов могут выступать различные динамические системы, при этом взаимодействующие осцилляторы могут иметь различную размерность фазового пространства [4].

На настоящий момент явление обобщенной синхронизации довольно подробно изучено на примере широкого круга взаимодействующих 
систем: исследованы как системы с дискретным временем, связанные однонаправленно и взаимно $[8,9]$, так и потоковые системы с однонаправленной $[10,11]$ и взаимной [5] связью (включая пространственнораспределенные системы [12]). Следующим этапом в исследовании явления обобщенной хаотической синхронизации стало рассмотрение сетей нелинейных элементов со сложной топологией связей [13], в частности, был изучен процесс установления режима обобщенной синхронизации при переходе от асинхронной динамики к синхронной в малой сети логистических отображений [14].

Для понимания природы хаотической синхронизации важную роль играют процессы установления синхронного режима при увеличении интенсивности связей между взаимодействующими системами. Известно, что переход от асинхронного поведения к синхронному режиму в двух связанных хаотических осцилляторах сопровождается перемежаемостью, когда при фиксированном значении параметра связи во временных реализациях рассматриваемых систем существуют участки синхронной динамики (ламинарные фазы), прерывающиеся участками асинхронного поведения (турбулентные фазы), при этом различным видам хаотической синхронизации соответствуют свои типы перемежаемости [15-18]. Для случая двух однонаправленно связанных осцилляторов известно, что переход к режиму обобщенной хаотической синхронизации сопровождается „оn-off“ перемежаемостью [17]. В то же самое время очевидно, что процесс установления режима обобщенной хаотической синхронизации в существенно более сложной системе, какой является сеть связанных осцилляторов, может характеризоваться другим сценарием, или, по крайней мере, другими характеристиками перемежающегося поведения, хотя не исключен вариант, что и в сетях со сложной топологией связей между элементами установление режима обобщенной хаотической синхронизации будет осуществляться через „on-of“ перемежаемость. В настоящее время этот вопрос остается полностью открытым.

Установление типа перемежающегося поведения основывается во многом на анализе статистических характеристик, таких как зависимость средней длительности ламинарных фаз от параметра надкритичности или распределение длительностей ламинарных участков поведения при фиксированных значениях управляющих параметров [19]. Однако для случая установления режима обобщенной хаотической синхронизации в сетях со сложной топологией связей выделить участ-

Письма в ЖТФ, 2017, том 43, вып. 7 
ки синхронной и асинхронной динамики с помощью традиционного метода [17], основанного на использовании вспомогательной системы [4], оказывается невозможным из-за того, что этот метод не может использоваться в случае наличия в системе взаимодействующих осцилляторов взаимных связей [5]. Соответственно для исследования процесса перехода к режиму обобщенной синхронизации в сети нелинейных осцилляторов ключевой задачей является создание и апробация метода выделения характерных участков синхронной и асинхронной динамики (ламинарных и турбулентных фаз) во временных реализациях взаимодействующих систем.

Таким образом, задачей данной работы является разработка метода выделения характерных фаз поведения в сложных сетях, находящихся вблизи границы режима обобщенной синхронизации.

Подход, предложенный для выделения ламинарных и турбулентных фаз динамики сети взаимодействующих осцилляторов, основан на методе ближайших соседей [2,20]. Рассмотрим два осциллятора изучаемой сети, например, с номерами $i$ и $j$. В фазовом пространстве одного из осцилляторов (к примеру, $i$-го) на аттракторе необходимо выбрать опорную точку $\mathbf{x}_{1}^{i}$, найти $N$ ближайших соседей $\left\{\mathbf{x}_{1 k}^{i}\right\}_{k=1, \ldots, N}$ для выбранной опорной точки, таких что $\left\|\mathbf{x}_{1}^{i}-\mathbf{x}_{1 k}^{i}\right\|<\delta$, и зафиксировать соответствующие им образы ближайших соседей $\mathbf{x}_{1}^{j i}$ и $\left\{\mathbf{x}_{1 k}^{j i}\right\}_{k=1, \ldots, N}$ в фазовом пространстве $j$-го осциллятора $[2,20]$. В качестве количественной характеристики степени синхронизма рассматриваемых $i$-го и $j$-го осцилляторов (в смысле обобщенной хаотической синхронизации) в опорной точке $\mathbf{x}_{1}^{i}$ следует рассматривать среднее значение $S$ расстояния между образами опорной точки и ближайших соседей в фазовом пространстве $j$-го осциллятора. Определяя величину

$$
S_{1}^{j i}=\frac{\sum_{k=1}^{N}\left\|\mathbf{x}_{1}^{j i}-\mathbf{x}_{1 k}^{j i}\right\|}{N}
$$

по соотношению значения $S_{1}^{j i}$ и некоторой наперед заданной пороговой величины $S_{c}$ (больше/меньше), можно определить характер динамики двух рассматриваемых узлов сети (синхронный/асинхронный) в момент времени, соответствующий нахождению $i$-й системы в выбранной опорной точке $\mathbf{x}_{1}^{i}$. Если теперь покрыть аттрактор $i$-го осциллятора большим количеством опорных точек $\left\{\mathbf{x}_{l}^{i}\right\}_{l=1, \ldots, M}$ с высокой плотностью покрытия, то, с помощью интерполяции, становится возможным 


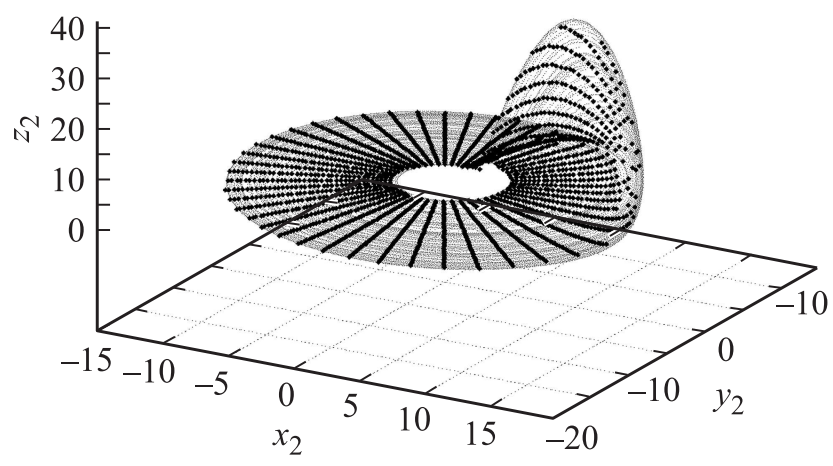

Рис. 1. Покрытие опорными точками $\left\{\mathbf{x}_{l}^{2}\right\}_{l=1, \ldots, M}$ хаотического аттрактора второго осциллятора.

получить представление о том, какой динамикой характеризуется взаимодействие выбранных осцилляторов сети в любой точке аттрактора. Такой подход позволяет оценить среднее расстояние между образами ближайших соседей $S(t)$ в каждый момент времени и соответственно определить текущую фазу динамики взаимодействия двух осцилляторов и выделить участки синхронной и асинхронной динамики для двух рассматриваемых узлов сети.

В качестве модельной системы для иллюстрации метода в данной работе была выбрана сеть связанных между собой потоковых систем, состоящая из $K=5$ взаимно связанных осцилляторов Ресслера. Эволюция $n$-го элемента сети $(n=1, \ldots, K)$ описывается следующей системой уравнений:

$$
\begin{gathered}
\frac{d x_{n}}{d t}=-\omega_{n} y_{n}-z_{n}+\varepsilon \sum_{n=1}^{K} C_{n m} x_{m}, \\
\frac{d y_{n}}{d t}=\omega_{n} x_{n}+a y_{n}, \\
\frac{d z_{n}}{d t}=p+z_{n}\left(x_{n}-c\right),
\end{gathered}
$$

где $a=0.15, p=0.2, c=10-$ управляющие параметры, $\varepsilon$ - параметр связи, $\mathbf{C}=\left\{C_{i j}\right\}-$ матрица, характеризующая топологию связей

Письма в ЖТФ, 2017, том 43, вып. 7 


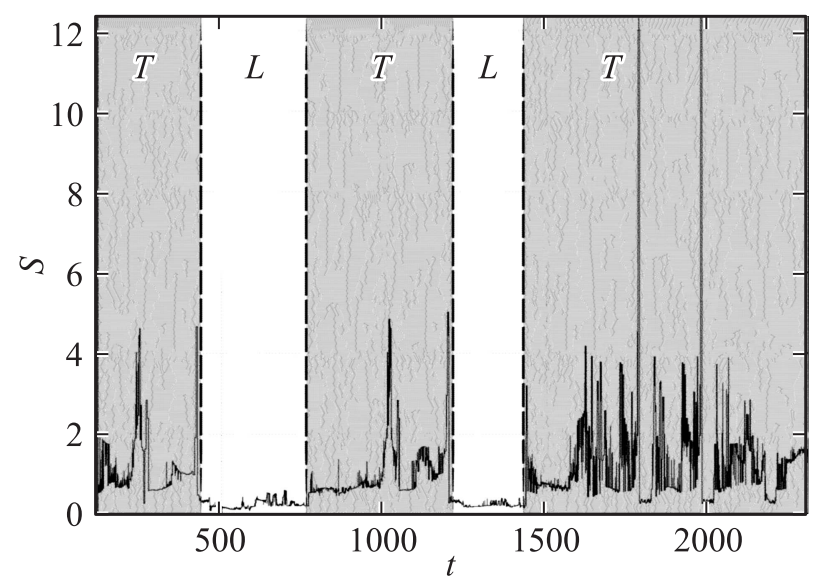

Рис. 2. Зависимость среднего расстояния $S(t)$ между образами ближайших соседей от времени $t$. Символами „ $T^{\prime}$ обозначены участки асинхронной динамики первого и второго осцилляторов („турбулентные фазы“), символами „L“ - участки синхронного поведения (,ламинарные фазы“).

между осцилляторами в сети $\left(C_{n m}=0-\right.$ отсутствие воздействия $m$-го элемента на $n$-й осциллятор, $C_{n m}=1-$ наличие воздействия $m$-го элемента на $n$-й осциллятор, $\left.C_{n n}=-\sum_{m \neq n} C_{n m}\right)$. Для обеспечения расстройки между взаимодействующими осцилляторами параметры, отвечающие за собственные частоты колебаний парциальных систем, были выбраны следующим образом: $\omega_{1}=0.95, \omega_{2}=0.9525, \omega_{3}=0.955$, $\omega_{4}=0.9575, \omega_{5}=0.96$. Тип связи между элементами сети был выбран двунаправленным в виде „каждый с каждым“, т. е. $C_{n m}=1(n \neq m)$. Для иллюстрации метода выбраны первый $(n=1)$ и второй $(n=2)$ узлы описанной сети. Во втором осцилляторе был задан массив опорных точек $\left\{\mathbf{x}_{l}^{2}\right\}_{l=1, \ldots, M}$, для которого в первом осцилляторе находились точки $\left\{\mathbf{x}_{l k}^{12}\right\}_{l=1, \ldots, M}$ и затем определялись значения $S_{l}^{12}$, с помощью которых, в свою очередь, интерполировалось значение $S(t)$.

На рис. 1 приведено покрытие опорными точками $\left\{\mathbf{x}_{l}^{2}\right\}_{l=1, \ldots, M}$ хаотического аттрактора второго осциллятора $(n=2)$. Информация о том, в каких областях хаотического аттрактора уже наблюдается синхронная динамика между рассматриваемыми осцилляторами, позволяет детекти- 
ровать начало и конец участка синхронного поведения рассматриваемых осцилляторов. На рис. 2 представлен фрагмент зависимости среднего расстояния $S(t)$ между образами ближайших соседей от времени $t$, где области „, $T^{6}$ соответствуют участкам асинхронной динамики, что определяется выполнением условия $S(t)>S_{c}$. Участки, обозначенные символом „ „L“, в свою очередь, соответствуют режиму синхронного поведения.

Таким образом, предлагаемый метод позволяет выделить характерные ламинарные и турбулентные участки поведения в системе со сложной перемежающейся динамикой, что в дальнейшем позволит осуществить анализ перехода к режиму обобщенной синхронизации в сети осцилляторов через перемежаемость.

Работа выполнена при поддержке Российского научного фонда (проект № 14-12-00224).

\section{Список литературы}

[1] Pikovsky A.S., Rosenblum M.G., Kurths J. Synchronization: A Universal Concept in Nonlinear Sciences. Cambridge University Press, 2001.

[2] Rulkov N.F., Sushchik M.M., Tsimring L.S., Abarbanel H.D.I. // Phys. Rev. E. 1995. V. 51. P. 980.

[3] Rulkov N.F. // Chaos. 1996. V. 6. P. 262.

[4] Abarbanel H.D.I., Rulkov N.F., Sushchik M.M. // Phys. Rev. E. 1996. V. 53. P. 4528.

[5] Moskalenko O.I., Koronovskii A.A., Hramov A.E. // Phys. Rev. E. 2013. V. 87. P. 64901.

[6] Zheng Z., Wang X., Cross M.C. // Phys. Rev. E. 2002. V. 65. P. 56211.

[7] Hung Y.-C., Huang Y.-T., Ho M.-C., Hu C.-K. // Phys. Rev. E. 2008. V. 77. P. 16202.

[8] Koronovskii A.A., Moskalenko O.I., Shurygina S.A., Hramov A.E. // Chaos. Solit. Fractals. 2013. V. 12.

[9] Pyragas K. // Phys. Rev. E. 1996. V. 54. P. R4508.

[10] Kocarev L., Parlitz U. // Phys. Rev. Lett. 1996. V. 76. P. 1816.

[11] Zheng Z., Hu G. // Phys. Rev. E. 2000. V. 62. P. 7882.

[12] Moskalenko O.I., Hramov A.E., Koronovskii A.A., Ovchinnikov A.A. // Europhys. J. B. 2011. V. 82. P. 69.

[13] Moskalenko O.I., Koronovskii A.A., Hramov A.E., Boccaletti S. // Phys. Rev. E. 2012. V. 86. P. 36216.

Письма в ЖТФ, 2017, том 43, вып. 7 
[14] Короновский А.А., Москаленко О.И., Пивоваров А.А., Храмов А.Е. // Письма в ЖТФ. 2015. V. 41. Р. 1.

[15] Rosenblum M.G., Pikovsky A.S., Kurths J. // Phys. Rev. Lett. 1997. V. 78. P. 4193.

[16] Boccaletti S., Valladares D.L. // Phys. Rev. E. 2000. V. 62. P. 7497.

[17] Hramov A.E., Koronovskii A.A. // Europhys. Lett. 2005. V. 70. P. 169.

[18] Boccaletti S., Allaria E., Meucci R., Arecchi F.T. // Phys. Rev. Lett. 2002. V. 89. P. 194101.

[19] Берже П., Помо И., Видаль К. Порядок в хаосе. М.: Мир, 1991.

[20] Parlitz U., Junge L., Lauterborn W., Kocarev L. // Phys. Rev. E. 1996. V. 54. P. 2115. 